\title{
MicroRNA-766 inhibits the malignant biological behaviours of pancreatic ductal adenocarcinoma by directly targeting ETS1
}

\author{
SHIQUAN LI, GUOQIANG YAN, MENG YUE, ZHENHUA KANG and LEI WANG \\ Department of Colorectal and Anal Surgery, The First Hospital of Jilin University, Changchun, Jilin 130021, P.R. China
}

Received April 17, 2018; Accepted November 13, 2018

DOI: $10.3892 / \mathrm{mmr} .2018 .9770$

\begin{abstract}
Increasing evidence indicates that numerous microRNAs (miRNAs) are altered in pancreatic ductal adenocarcinoma (PDAC), and their alterations significantly influence the malignant behaviour of PDAC. Therefore, identifying miRNAs associated with PDAC and their biological roles in the disease may provide promising therapeutic opportunities. Alteration of the expression of miRNA-766 (miR-766) has been previously reported in several types of human malignancy. However, to the best of our knowledge, whether miR-766 exhibits different expression patterns in PDAC and its underlying functions in the progression of PDAC remain to be elucidated. In the present study, reverse transcription-quantitative polymerase chain reaction (RT-qPCR) was used to detect miR-766 expression levels in PDAC tissues and cell lines. The effects of miR-766 upregulation on PDAC cell proliferation and invasion were evaluated using MTT and invasion assays, respectively. The mechanisms underlying the role of miR-766 in PDAC cells were explored using bioinformatics analysis, luciferase reporter assay, RT-qPCR and western blot analysis. It was found that miR-766 was significantly downregulated in PDAC tissues and cell lines. The detailed roles of miR-766 in the progression of PDAC were characterised using Panc-1 and Aspc-1 cell lines. The results revealed that the upregulation of miR-766 restricted the proliferation and invasion of PDAC cells. Through a series of experiments, it was found that E26 transformation specific-1 (ETS1) was a direct target of miR-766 in PDAC cells. Furthermore, ETS1 knockdown simulated the inhibitory effects of the overexpression of miR-766 on PDAC cells, whereas the effects of miR-766 restoration on the PDAC cells were reversed by overexpressing ETS1. In conclusion, the findings of the present study demonstrate that miR-766 offers potential as a therapeutic target for patients with PDAC.
\end{abstract}

Correspondence to: Professor Lei Wang, Department of Colorectal and Anal Surgery, The First Hospital of Jilin University, 71 Xinmin Road, Changchun, Jilin 130021, P.R. China

E-mail: wang_lei1967@126.com

Key words: pancreatic ductal adenocarcinoma, microRNA-766, E26 transformation specific-1, proliferation, invasion

\section{Introduction}

Pancreatic cancer is one of the most life-threatening malignant tumours worldwide (1). Pancreatic ductal adenocarcinoma (PDAC) is the primary type of pancreatic cancer and accounts for $\sim 96 \%$ of all cases of the disease (2). Despite the substantial progress made in therapeutic techniques, including surgery and radiochemotherapy, the therapeutic outcomes of patients with PDAC remain unsatisfactory (3). The poor prognosis of these patients is mainly attributed to the lack of an effective early diagnostic strategy and the aggressive characteristics of PDAC, which include rapid growth and early metastasis (4). The overall five-year-survival rates of patients with PDAC are $<5 \%$ (5), whereas those in patients who receive surgical resection range between 10 and $25 \%$ (6). Therefore, a comprehensive understanding of the mechanisms responsible for the occurrence and development of PDAC is required for the identification of novel diagnostic biomarkers and promising therapeutic methods for treating patients with this life-threatening disease.

MicroRNAs (miRNAs) are a group of endogenous, short, non-coding RNA molecules composed of 19-25 nucleotides. They are vital gene expression regulators and can completely or incompletely interact with the 3'-untranslated regions (3'-UTRs) of their target messenger RNAs (mRNAs) to prevent mRNA translation and/or promote mRNA degradation (7). Recently, several miRNAs have been identified to be differently expressed in PDAC, including miRNA (miR)-7-5p (8), miR-410-3p (9), miR-212 (10) and miR-454 (11). The aberrant expression of miRNAs is closely associated with the development and malignant progression of PDAC and serve as oncogenes or tumour suppressors $(12,13)$. miRNAs regulate the multiple biological behaviours of PDAC cells, including cell proliferation, apoptosis, cell cycle, lymphangiogenesis, invasion, metastasis, epithelial-mesenchymal transition and chemotherapeutic resistance $(9,11,14-16)$. Therefore, miRNAs are potential diagnostic biomarkers and therapeutic targets for patients with PDAC, and the association between miRNAs and PDAC requires further clarification.

Alteration in the expression of miR-766 has been previously reported in several types of human malignancy (17-19). However, to the best of our knowledge, whether miR-766 exhibits different expression patterns in PDAC and its underlying functions in the progression of PDAC remain to be elucidated. Therefore, the present study aimed to detect the 
expression of miR-766 in PDAC tissues and cell lines, characterise the role of miR-766 in PDAC cells and investigate the mechanism underlying the roles of miR-766 in PDAC. An improved understanding of miR-766 in PDAC may be useful in identifying therapeutic targets for the treatment of patients with this aggressive malignant tumour.

\section{Materials and methods}

Sample collection. In total, 29 pairs of PDAC tissues and matched normal adjacent tissues (NATs) were collected from The First Hospital of Jilin University (Jilin, China) between June 2015 and March 2017. None of the patients (16 men, 13 women; age range, 39-64 years) had been treated with chemotherapy, radiotherapy or other treatments prior to surgical excision. All tissue specimens were immediately snap-frozen in liquid nitrogen and then stored at $-80^{\circ} \mathrm{C}$ until further RNA isolation. The study was approved by the Clinical Research Ethics Committee of The First Hospital of Jilin University. Written informed consent was also obtained from all participants.

Cell culture and transfection. The HPDE6c7 normal human pancreatic cell line was ordered from American Type Culture Collection (Manassas, VA, USA). A total of three human PDAC cell lines, including Panc-1, Aspc-1 and Sw1990, were purchased from the Chinese Cell Bank of the Chinese Academy of Sciences (Shanghai, China). All cell lines were cultured in Dulbecco's modified Eagle's medium (DMEM) supplemented with $10 \%$ heat inactivated fetal bovine serum (FBS) and 1\% penicillin/streptomycin mixture (all from Gibco; Thermo Fisher Scientific, Inc., Waltham, MA, USA). The cells were incubated in a cell incubator containing 5\% $\mathrm{CO}_{2}$ at $37^{\circ} \mathrm{C}$.

miR-766 mimics and negative control miRNA mimics (miR-NC) were purchased from GenePharma (Shanghai, China). A small interfering RNA (siRNA) against the expression of E26 transformation specific-1 (ETS1), termed si-ETS1 (5'-ACUUGCUACCAUCCCGUACTT-3'), and negative control siRNA (si-NC; 5'-UUCUCCGAACGUGUCACGUTT-3') were purchased from RiboBio Co., Ltd. (Guangzhou, China). The ETS1 overexpression vector pCMV-ETS1 and empty pCMV plasmid were purchased from Amspring Biological Technology Co., Ltd. (Changsha, China). The cells were plated into 6 -well plates with a density of $5 \times 10^{5} 1$ day prior to transfection. The cells were transfected with miRNA mimics, siRNA or vector using Lipofectamine 2000 (Invitrogen; Thermo Fisher Scientific, Inc.) according to the manufacturer's protocol. The transfected cells were subsequently incubated at $37^{\circ} \mathrm{C}$ with $5 \% \mathrm{CO}_{2}$. Reverse transcription-quantitative polymerase chain reaction (RT-qPCR) and western blot analyses were employed to determine the transfection efficiency.

RT-qPCR analysis. Total RNA was isolated from tissues or cells using TRIzol reagent (Thermo Fisher Scientific, Inc.). A NanoDrop Spectrophotometer (NanoDrop Technologies; Thermo Fisher Scientific, Inc.) was utilised to determine the concentration of total RNA. Synthesis of miR-766 complementary DNA (cDNA) was performed using a TaqMan MicroRNA Reverse Transcription kit (Applied Biosystems;
Thermo Fisher Scientific, Inc.). Subsequently, the complementary DNA (cDNA) was subjected to qPCR analysis using a TaqMan MicroRNA qPCR Assay kit (Applied Biosystems; Thermo Fisher Scientific, Inc.). For the quantification of ETS1 mRNA, total RNA was reversed transcribed into cDNA with a PrimeScript $^{\mathrm{TM}}$ RT Master mix (Takara Bio, Inc., Otsu, Japan). PCR amplification was performed using SYBR Green PCR master mix (Takara Bio, Inc.), according to the manufacturer's protocol. The thermocycling conditions were as follows: $95^{\circ} \mathrm{C}$ for $10 \mathrm{~min}$, followed by 40 cycles of $95^{\circ} \mathrm{C}$ for $15 \mathrm{sec}$ and $60^{\circ} \mathrm{C}$ for $1 \mathrm{~min}$. Relative mRNA levels of miR-766 and ETS1 were analysed based on the $2^{-\Delta \Delta \mathrm{Cq}}$ method (20) and normalised by U6 snRNA or GAPDH, respectively.

\section{3-(4,5-dimethyl-2-thiazolyl)-2,5-diphenyl-2-H-tetrazolium} bromide (MTT) assay. The transfected cells were collected at $24 \mathrm{~h}$ post-transfection and were reseeded into 96-well plates at a density of $2 \times 10^{3}$ cells in each well. The culture plates were then incubated at $37^{\circ} \mathrm{C}$ with $5 \% \mathrm{CO}_{2}$ for $0,24,48$ and $72 \mathrm{~h}$. The MTT assay was performed at every time point to detect cell proliferation. In detail, $20 \mu \mathrm{l}$ of MTT solution $(5 \mathrm{mg} / \mathrm{ml}$; Beyotime Institute of Biotechnology, Shanghai China) was added into each well, and the cells were incubated at $37^{\circ} \mathrm{C}$ with $5 \% \mathrm{CO}_{2}$ for an additional $4 \mathrm{~h}$. Subsequently, the culture supernatant was gently removed, following which $150 \mu \mathrm{l}$ of dimethyl sulfoxide (Beyotime Institute of Biotechnology) was added into each well. Following further incubation at $37^{\circ} \mathrm{C}$ for $15 \mathrm{~min}$, the absorbance at a wavelength of $490 \mathrm{~nm}$ was detected on a microplate reader (Bio-Rad Laboratories, Inc., Hercules, CA, USA).

Invasion assay. The invasive ability of the PDAC cells was assessed using 24-well Transwell chambers ( $8-\mu \mathrm{m}$ pore size) coated with Matrigel (both from BD Biosciences, Franklin Lakes, NJ, USA). The transfected cells were suspended in FBS-free DMEM and then added into the upper compartment of each chamber with an initial density of $1 \times 10^{5}$ cells/well. The lower compartments were covered with $500 \mu$ l DMEM containing 20\% FBS. Following culture for $24 \mathrm{~h}$ at $37^{\circ} \mathrm{C}$, the cells remaining on the upper surface of the Transwell chambers were gently removed by wiping with cotton-tipped swabs. The invasive cells were fixed with methanol, stained with $0.1 \%$ crystal violet and images were captured under an optical microscope (Olympus Corporation, Tokyo, Japan). A total of five fields per chamber (x200 magnification) were randomly selected, and the average number of invasive cells was counted.

Bioinformatics analysis and luciferase reporter assay. The potential targets of miR-766 were predicted using TargetScan (http://www.targetscan.org/) and microrna.org (http://www. microrna.org/). ETS1 was predicted as a major candidate of miR-766. Luciferase reporter plasmids were constructed to examine whether ETS1 was a target gene of miR-766. The 3'-UTR fragment of ETS1 containing the putative wild-type (wt) or mutant (mut) binding site were chemically produced by GenePharma, and were inserted into pGL3 firefly luciferase reporter vector (Promega Corporation, Madison, WI, USA). The synthesised luciferase reporter plasmids were termed pGL3-ETS1-3'-UTR wt and pGL3-ETS1-3'-UTR mut, 
respectively. The miR-766 mimics or miR-NC was co-transfected with pGL3-ETS1-3'-UTR wt or pGL3-ETS1-3'-UTR mut into cells using Lipofectamine 2000, in accordance with the manufacturer's protocols. After $48 \mathrm{~h}$ of transfection, the transfected cells were harvested and luciferase activities were detected using the Dual-Luciferase Reporter Assay System (Promega Corporation). Renilla luciferase activity was normalised to firefly luciferase activity.

Western blot analysis. Total protein of the tissues or cells was lysed using radioimmunoprecipitation assay lysis buffer, and the concentration of total protein was determined using Bicinchoninic Acid kit (both from Sigma; Merck KGaA, Darmstadt, Germany). Equal quantities of protein $(30 \mu \mathrm{g})$ were separated by $10 \%$ SDS-polyacrylamide gel electrophoresis and transferred onto polyvinylidene fluoride membranes (EMD Millipore, Billerica, MA, USA). The membranes were then blocked with 5\% nonfat milk in Tris-buffered saline containing $0.1 \%$ Tween-20 (TBST) for $2 \mathrm{~h}$ at room temperature, followed by incubation overnight at $4^{\circ} \mathrm{C}$ with the following primary antibodies: Mouse anti-human monoclonal ETS1 (1:1,000 dilution; cat no. sc-55581; Santa Cruz Biotechnology, Inc., Dallas, TX, USA) and mouse anti-human monoclonal GAPDH (1:1,000 dilution; cat no. sc-32233; Santa Cruz Biotechnology, Inc.). Following washing three times with TBST, the membranes were incubated with goat anti-mouse horseradish peroxidase-conjugated secondary antibody (1:5,000 dilution; cat no. sc-516102; Santa Cruz Biotechnology, Inc.) at room temperature for $2 \mathrm{~h}$. Finally, the protein signals were visualised using an enhanced chemiluminescence reagent (GE Healthcare Life Sciences, Little Chalfont, UK). Protein expression levels were analyzed using Quantity One ${ }^{\circledR}$ software version 4.62 (Bio-Rad Laboratories, Inc.). GAPDH served as an internal control for the protein expression of ETS1.

Statistical analysis. Data are presented as the median \pm standard deviation. SPSS version 19.0 software (IBM Corp., Armonk, NY, USA) was used to analyse all data. Comparisons among groups were made with Student's t-test and one-way analysis (ANOVA) of variance for multiple comparisons. The Student-Newman-Keuls test was used as a post hoc test following ANOVA. The association between miR-766 and ETS1 mRNA levels in PDAC tissues was evaluated using Spearman's correlation analysis. $\mathrm{P}<0.05$ was considered to indicate a statistically significant difference.

\section{Results}

miR-766 levels are reduced in human PDAC tissues and cell lines. The differential expression of miR-766 has been reported in several types of human cancer (17-19). However, whether miR-766 is involved in the progression of PDAC remains unclear. Therefore, RT-qPCR analysis was performed for the detection of the expression of miR-766 in 29 pairs of PDAC tissues and matched NATs. The results revealed that the level of miR-766 was lower in PDAC tissues than that in NATs $(\mathrm{P}<0.05$; Fig. 1A). The expression level of miR-766 was also determined in three PDAC cell lines (Panc-1, Aspc-1 and Sw1990) and the normal human pancreatic HPDE6c7 cell line. The results of the RT-qPCR analysis indicated that miR-766
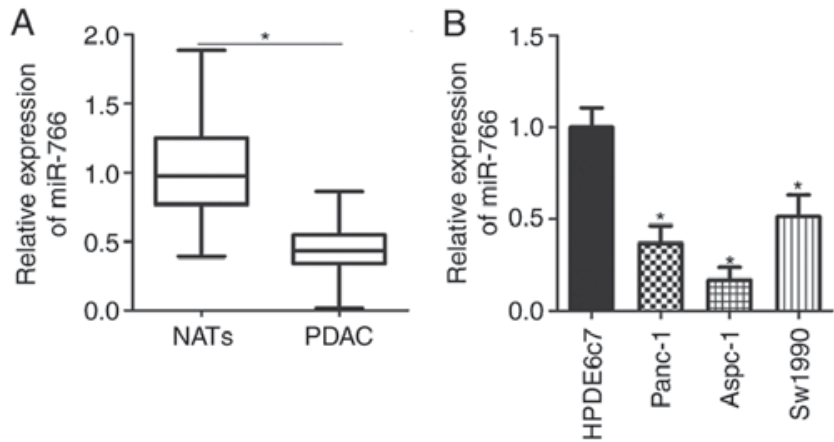

Figure 1. Reduced expression levels of miR-766 are observed in PDAC tissues and cell lines. (A) Reverse transcription-quantitative polymerase chain reaction analysis was used to examine the expression of miR-766in 29 pairs of PDAC tissues and matched NATs. * $\mathrm{P}<0.05$ vs. NATs. (B) Expression of miR-766 was analysed in three PDAC cell lines (Panc-1, Aspc-1 and Sw1990) and a normal human pancreatic cell line (HPDE6c7). ${ }^{*} \mathrm{P}<0.05$ vs. HPDE6c7. PDAC, pancreatic ductal adenocarcinoma; NATs, normal adjacent tissues; miR, microRNA.

was underexpressed in all three PDAC cell lines compared with that in HPDE6c7 cells $(\mathrm{P}<0.05$; Fig. 1B). These results indicate that the decreased expression of miR-766 may be crucial in the progression of PDAC.

miR-766 inhibits the proliferation and invasion of PDAC cells in vitro. To analyse the detailed roles of miR-766 in PDAC, miR-766 mimics or miR-NC were introduced into two PDAC cell lines, namely, Panc-1 and Aspc-1, which exhibited the lowest expression of miR-766 amongst the three PDAC cell lines. The transfection of miR-766 mimics markedly increased the expression of miR-766 in Panc-1 and Aspc-1 cells compared with those in cells transfected with miR-NC $(\mathrm{P}<0.05$; Fig. 2A). MTT assays were performed to examine whether the upregulation of miR-766 inhibited PDAC cell proliferation. The overexpression of miR-766 caused a significant reduction in the proliferation rates of the Panc-1 and Aspc- 1 cells $(\mathrm{P}<0.05 ;$ Fig. 2B). Invasion assays were then conducted to determine whether the exogenous expression of miR-766 affected the invasive capacities of the PDAC cells. A marked decrease in cell invasion ability was observed in the miR-766-overexpressing Panc-1 and Aspc-1 cells $(\mathrm{P}<0.05$; Fig. 2C). Overall, these data demonstrate that miR-766 inhibits the proliferative and invasive abilities of PDAC cells.

miR-766 inhibits the expression of ETS1 by directly targeting its 3 '-UTR. miRNAs exert their effects by directly targeting and inhibiting the expression of their target genes. Therefore, the targets of miR-766 in PDAC cells were identified. Bioinformatics analysis was used for the prediction of the putative targets of miR-766. According to the analysis, ETS1 was identified as a major target of miR-766, and the 3'-UTR of ETS1 contains an match binding sequence in the seed region of miR-766 (Fig. 3A). Whether miR-766 directly targets the 3'-UTR region of ETS1 was determined by constructing luciferase reporter plasmids and using them for luciferase reporter assays. As shown in Fig. 3B, the restoration of miR-766 significantly decreased the luciferase activities of the plasmid carrying the wt ETS1 3'-UTR in Panc-1 and Aspc-1 cells $(\mathrm{P}<0.05)$. However, the luciferase activities of the 

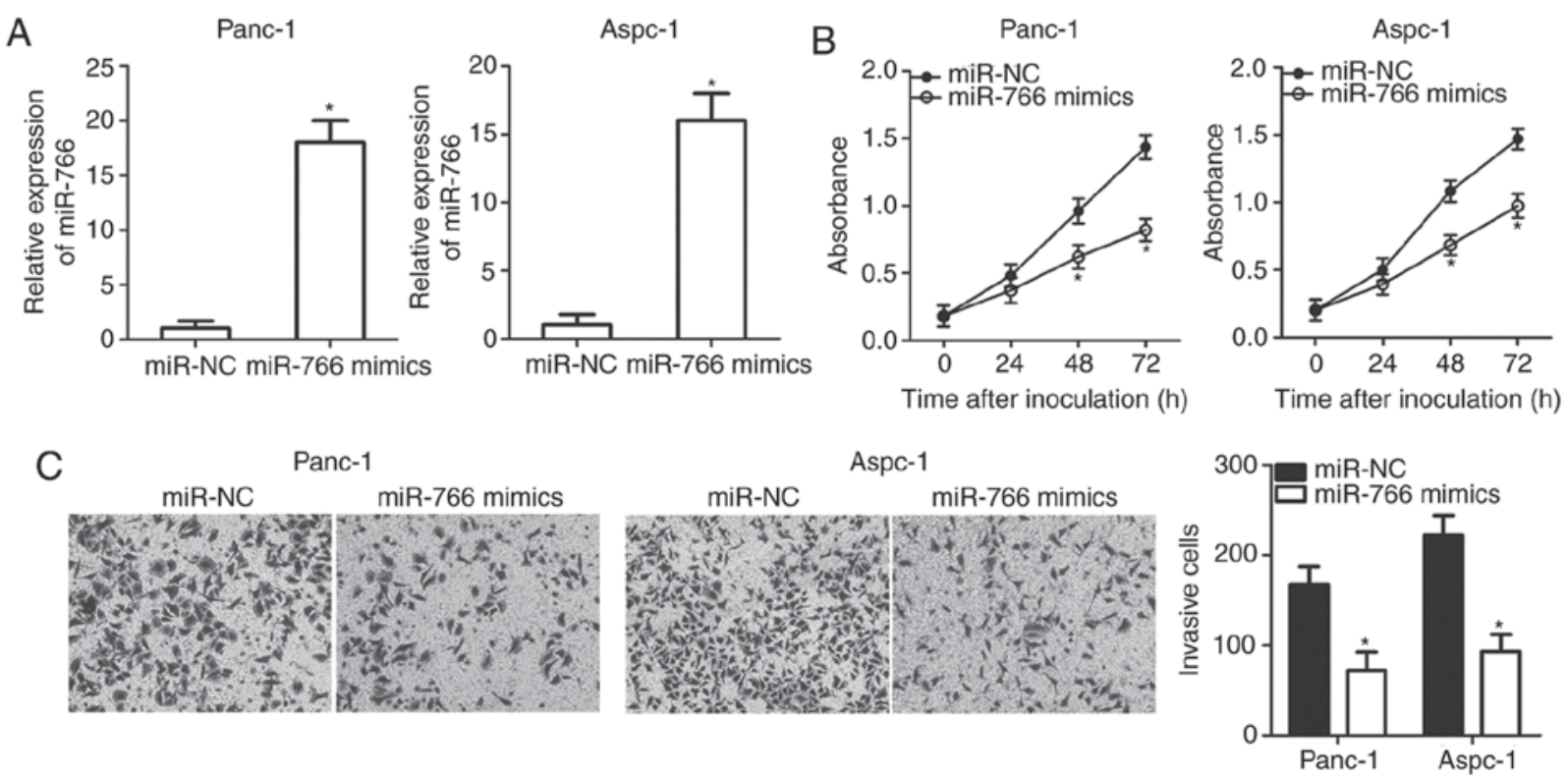

Figure 2. Overexpression of miR-766 attenuates the proliferation and invasion of Panc-1 and Aspc-1 cells. (A) Panc-1 and Aspc- 1 cells were treated with miR-766 mimics or miR-NC. Reverse transcription-quantitative polymerase chain reaction analysis was performed at $48 \mathrm{~h}$ post-transfection to determine the expression of miR-766. "P<0.05 vs. miR-NC. (B) Proliferation of Panc-1 and Aspc-1 cells transfected with miR-766 mimics or miR-NC was detected using a 3-(4,5-dimethyl-2-thiazolyl)-2,5-diphenyl-2-H-tetrazolium bromide assay. "P<0.05 vs. miR-NC. (C) Effect of the overexpression of miR-766 on Panc-1 and Aspc-1 cell invasion was determined using invasion assay (x200 magnification). ${ }^{*} \mathrm{P}<0.05$ vs. miR-NC. miR, microRNA; NC, negative control.

plasmid harbouring the mut ETS1 3'-UTR were unchanged. Subsequently, RT-qPCR and western blot analyses were performed for detection of the expression of ETS1 in the Panc-1 and Aspc-1 cells transfected with miR-766 mimics or miR-NC. As expected, the mRNA ( $<<0.05$; Fig. $3 \mathrm{C})$ and protein $(\mathrm{P}<0.05$; Fig. 3D) levels of ETS1 were reduced upon ectopic overexpression of miR-766 in the Panc-1 and Aspc-1 cells. Collectively, these results provide evidence that ETS1 is a direct target gene of miR-766 in PDAC cells.

Inhibition of ETS1 can simulate the suppressive roles of miR-766 in PDAC cells. Specific si-ETS1 was used to knock down the endogenous expression of ETS1 in the Panc-1 and Aspc-1 cells for the evaluation of the roles of ETS1 in PDAC $(\mathrm{P}<0.05 ;$ Fig. 4A). The results of the MTT assays revealed that ETS1 knockdown suppressed the proliferation of the Panc-1 and Aspc-1 cells compared with that in the si-NC groups (Fig. 4B and $\mathrm{C}, \mathrm{P}<0.05$ ). The effect of the downregulation of ETS1 on PDAC cell invasion was then investigated. The silencing of ETS1 effectively restricted the invasion ability of Panc-1 and Aspc-1 cells ( $\mathrm{P}<0.05$; Fig. 4D). These results demonstrate that the effects of the inhibition of ETS1 on PDAC cells are similar to those induced by the overexpression of miR-766, suggesting that ETS1 is a functional target of miR-766 in PDAC cells.

miR-766 restricts the proliferation and invasion of PDAC cells via downregulating ETS1. Considering the aforementioned results, the present study examined whether ETS1 mediates the suppressive effects of the overexpression of miR-766 on PDAC cell proliferation and invasion. The Panc-1 and Aspc-1 cells were co-transfected with miR-766 mimics and the empty pCMV plasmid or the pCMV-ETS1ETS1 overexpression vector. As shown in Fig. 5A, the protein levels of ETS1 were restored in the miR-766 mimic-transfected Panc-1 and Aspc-1 cells following co-transfection with pCMV-ETS1 $(\mathrm{P}<0.05)$. Furthermore, the overexpression of ETS1 reversed the inhibitory effects of miR-766 mimics on the proliferation $(\mathrm{P}<0.05$; Fig. 5B and $\mathrm{C})$ and invasion $(\mathrm{P}<0.05$; Fig. 5D) of Panc- 1 and Aspc-1 cells. These results further confirmed that miR-766 inhibits PDAC cell proliferation and invasion by inhibiting the expression of ETS1.

\section{Discussion}

Increasing evidence indicates that numerous miRNAs are altered in PDAC, and their alterations have significant effects on the malignant behaviour of PDAC (21-23). Therefore, identifying miRNAs associated with PDAC and their biological roles in the disease may provide promising therapeutic opportunities. In the present study, it was demonstrated that the expression of miR-766 was weak in PDAC tissues and cell lines. The ectopic expression of miR-766 impeded cell proliferation and invasion in PDAC, and ETS1 was found to be a direct target gene of miR-766 in PDAC cells. Overall, these findings suggest that miR-766 inhibits the development of PDAC by directly targeting ETS1.

miR-766 exhibits different expression patterns in several types of human cancer. For example, miR-766 is significantly downregulated in renal cell carcinoma tissues and cell lines. The reduced expression of miR-766 exhibits a clear association with the clinical stage of renal cell carcinoma. Furthermore, patients with renal cell carcinoma and low levels of miR-766 have poorer prognosis than those with a high level of miR-766 (17). However, the expression of miR-766 is upregulated in colorectal cancer (18) and lung adenocarcinoma (19), and miR-766 is an independent prognostic marker of overall survival rate for patients with lung adenocarcinoma (19). 


\section{A ETS1-3'-UTR wt \\ 5'...CUGGCACAUCAUUUUGCUGGAGA... $3^{\prime}$ $3^{\prime}$ CGACUCCGACACCCCGACCUCA $5^{\prime}$ hsa-miR-766 \\ 5'...CUGGCACAUCAUUUU CGACCUCA... $3^{\prime}$ ETS1-3'-UTR mut}
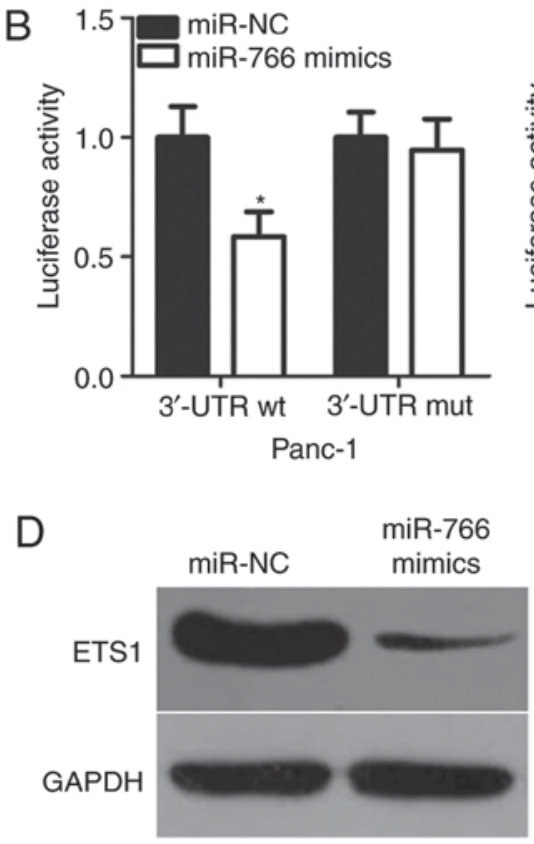

Panc-1
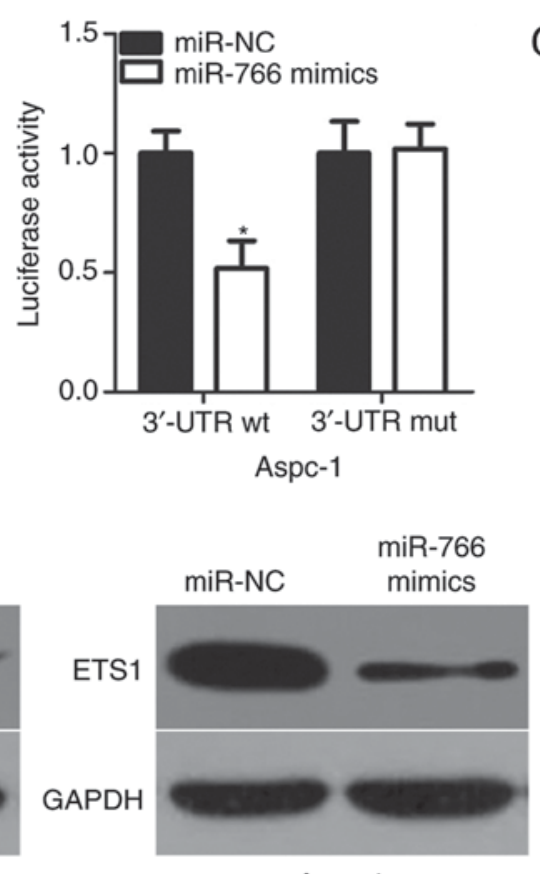

Aspc-1
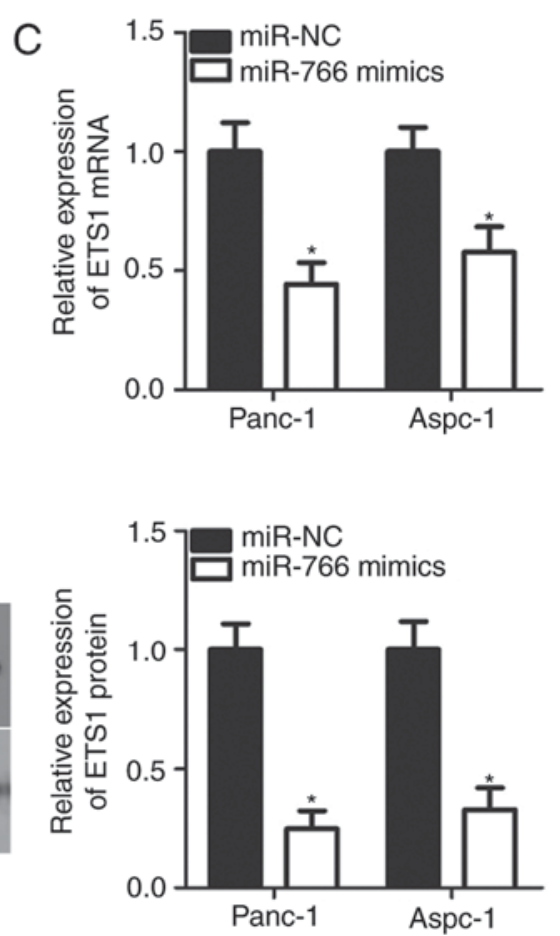

Figure 3. miR-766 directly targets ETS1 in pancreatic ductal adenocarcinoma cells. (A) Predicted miR-766 binding sequences within the 3'-UTR of ETS1 and a mutated version generated by site directed mutagenesis are shown. "P $<0.05$ vs. miR-NC. (B) Panc-1 and Aspc-1 cells were co-transfected with pGL3-ETS1-3'-UTR wt or pGL3-ETS1-3'-UTR mut, and miR-766 mimics or miR-NC. Luciferase activity was detected at $48 \mathrm{~h}$ post-transfection. ${ }^{*} \mathrm{P}<0.05 \mathrm{vs}$. miR-NC. The (C) mRNA and (D) protein expression of ETS1 was determined in Panc-1 and Aspc-1 cells that were transfected with miR-766 mimics or miR-NC. "P<0.05 vs. miR-NC. miR, microRNA; NC, negative control; ETS1, E26 transformation specific-1; 3'-UTR, 3'-untranslated region; wt, wild-type; mut, mutant.

A

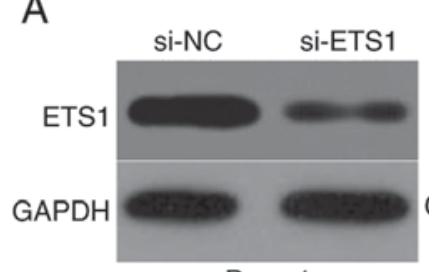

Panc-1

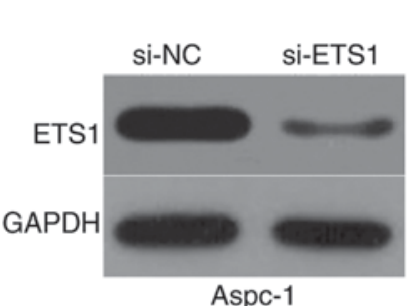

Aspc-1

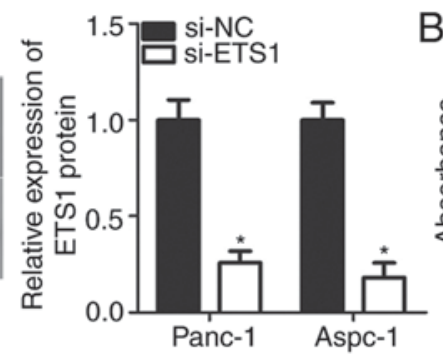

B $\quad 2.07-$ si-NC

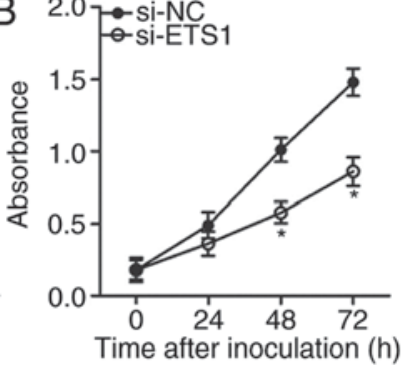

Panc-1

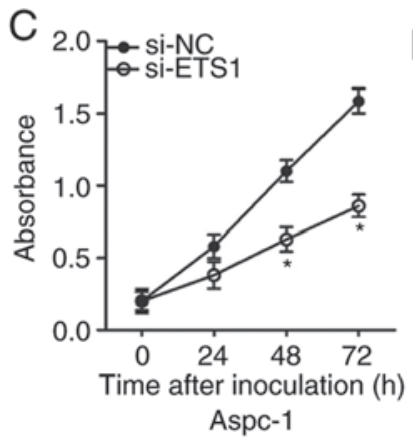

D

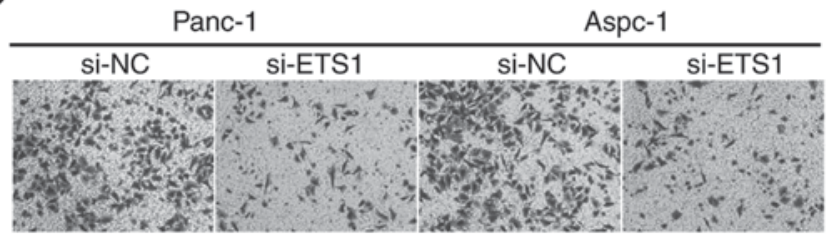

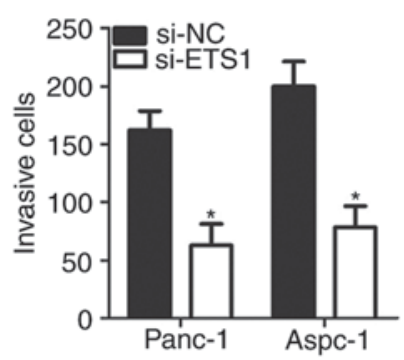

Figure 4. Inhibition of ETS1 restricts Panc-1 and Aspc-1 cell proliferation and invasion. (A) Panc-1 and Aspc-1 cells were transfected with siRNA against ETS1 (si-ETS1) to knock down the endogenous expression of ETS1. "P $<0.05$ vs. si-NC. The proliferative abilities of (B) Panc-1 and (C) Aspc-1 cells, and their (D) invasive abilities were evaluated using 3-(4,5-dimethyl-2-thiazolyl)-2,5-diphenyl-2-H-tetrazolium bromide and invasion assays, respectively. " $\mathrm{P}<0.05$ vs. si-NC. ETS1, E26 transformation specific-1; siRNA, small interfering RNA; NC, negative control. 
A
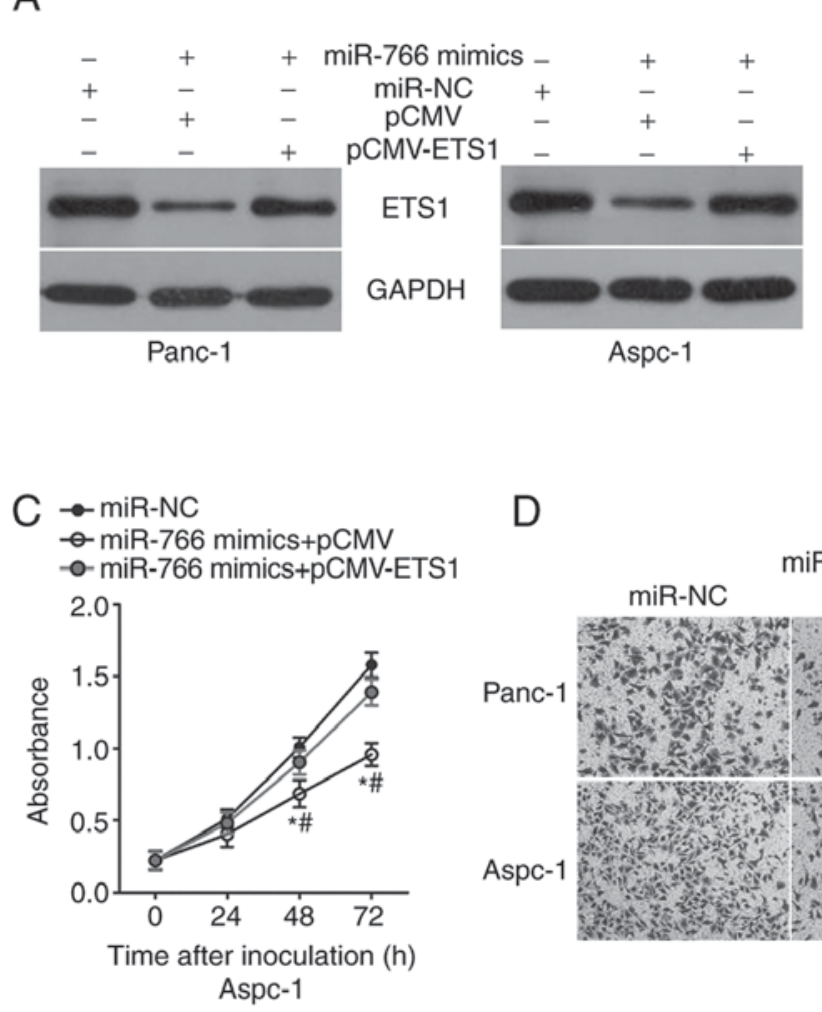

D
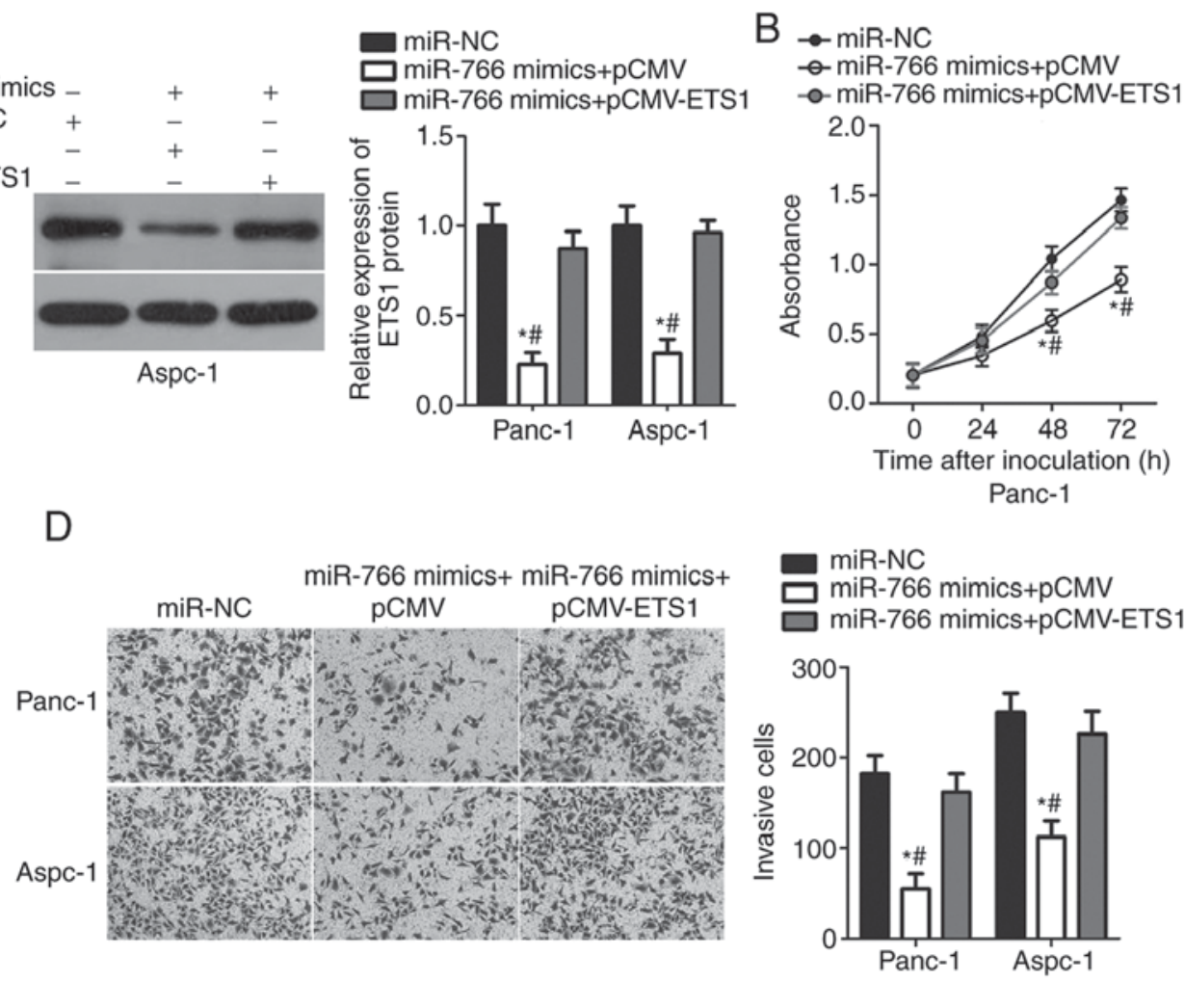
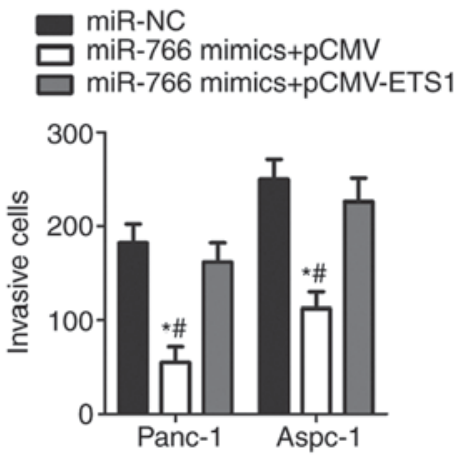

Figure 5. Inhibition of ETS1 mediates the suppressive effects of miR-766 on PDAC cells. (A) Protein expression of ETS1 in Panc-1 and Aspc-1 cells was analysed by western blot analysis following transfection with miR-766 mimics in addition to pCMV or pCMV-ETS1. "P<0.05 vs. miR-NC; ${ }^{*} \mathrm{P}<0.05$ vs. miR-766 mimics + pCMV-ETS1. 3-(4,5-dimethyl-2-thiazolyl)-2,5-diphenyl-2-H-tetrazolium bromide and invasion assays (x200 magnification) were used to determine the proliferation of (B) Panc-1 and (C) Aspc-1 cells and their (D) invasion following co-transfection with miR-766 mimics and pCMV or pCMV-ETS1. ${ }^{*}<0.05$ vs. miR-NC; "P<0.05 vs. miR-766 mimics + pCMV-ETS1. ETS1, E26 transformation specific-1; miR, microRNA; NC, negative control.

These conflicting studies indicate that the expression pattern of miR-766 exhibits tissue specificity. These findings also suggest that this miRNA is an effective biomarker for the diagnosis and prediction of the therapeutic outcomes of patients with these types of cancer.

miR-766 has been demonstrated to be closely linked to the initiation and progression of several types of cancer in humans. For example, the restoration of miR-766 attenuates renal cell carcinoma cell proliferation and colony formation, induces cell cycle arrest in vitro and reduces tumour growth in vivo (17). The enforced expression of miR-766 decreases tumour sphere formation and invasion in vitro and reduces in vivo lung metastasis in breast cancer (24). However, miR-766 has an oncogenic role in colorectal cancer by promoting cell growth and anchorage-independent growth $(18,25)$. These findings suggest that miR-766 should be developed as a future therapeutic target for effectively controlling the progression of these types of cancer in humans

Several genes, including splicing factor SF2 (17) in renal cell carcinoma, and sex determining region Y-box 6 (18) and DNA methyltransferase 3B (25) in colorectal cancer, are direct targets of miR-766. In the present study, the direct target gene of miR-766 was investigated to elucidate the mechanism underlying the effect of miR-766 in PDAC. Firstly, ETS1 was predicted as a major target of miR-766 using bioinformatics analysis. Secondly, the results of the luciferase reporter assays demonstrated that miR-766 directly recognises and binds to the 3'-UTR of ETS1. Thirdly, the overexpression of miR-766 decreased the expression of ETS1 at the mRNA and protein levels in PDAC cells. Finally, the inhibition of ETS1 resulted in the same effects as the overexpression of miR-766 on the proliferation and invasion of PDAC cells. Notably, the reintroduction of ETS1 abrogated the tumour-suppressing effects of miR-766 in PDAC cells. These findings sufficiently proved that ETS1 is a direct and downstream functional target of miR-766 in PDAC cells. However, the present study was unable not conclude that ETS1 was the primary or only target of miR-766 in PDAC. Furthermore, a previous study demonstrated that the 3'-UTR of EST1 was recognised by other genes (26), suggesting that miR-766 may also indirectly influence the activity of ETS1 by targeting genes that regulate ETS1.

ETS1 is a member of the ETS family of transcription factors and is upregulated in PDAC. The upregulation of ETS1 is correlated with the differentiation of PDAC (27). Functionally, ETS1 has an oncogenic role in the occurrence and development of PDAC by affecting cell proliferation, angiogenesis, chemotherapeutic chemoresistance, epithelial-mesenchymal transition and motility (28-31). miRNA-based targeted therapy that inhibits or restores expression is a potential therapeutic technique for anticancer therapy (32). Given the crucial roles of ETS1 in PDAC, an miR-766-based targeted therapy against the expression of ETS1 may provide novel and efficient therapeutic opportunities for the management of patients with PDAC.

In conclusion, the expression of miR-766 was reduced in PDAC tissues and cell lines in the present study. The 
upregulation of miR-766 suppressed PDAC cell proliferation and invasion by directly targeting ETS1. The results revealed that the miR-766/ETS1 pathway may explain the complex mechanisms that regulate the oncogenesis and development of PDAC and represents a novel approach for treating patients with PDAC. However, the present study did not analyse the association between miR-766 and the clinical features of patients with PDAC. This limitation is to be resolved in future experiments. In addition, future investigations aim to detect the mRNA levels of ETS1 and investigate the association between miR-766 and ETS1 mRNA levels in PDAC tissues.

\section{Acknowledgements}

Not applicable.

\section{Funding}

No funding was received.

\section{Availability of data and materials}

The datasets used and/or analysed during the present study are available from the corresponding author upon reasonable request.

\section{Authors' contributions}

All authors have made a significant contribution to the findings and methods. LW and SL designed the study. SL, GY, MY and ZK performed the functional experiments. The authors have read and approved the final draft.

\section{Ethics approval and consent to participate}

The present study was approved by The First Hospital of Jilin University, and was performed in accordance with the Declaration of Helsinki and the guidelines of the Ethics Committee of The First Hospital of Jilin University. Written informed consent was obtained from all patients for the use of their clinical tissues.

\section{Patient consent for publication}

Not applicable.

\section{Competing interests}

The authors declare that they have no competing interests.

\section{References}

1. Siegel R, Ma J, Zou Z and Jemal A: Cancer statistics, 2014. CA Cancer J Clin 64: 9-29, 2014

2. Modolell I, Guarner L and Malagelada JR: Vagaries of clinical presentation of pancreatic and biliary tract cancer. Ann Oncol 10 (Suppl 4): S82-S84, 1999.

3. Hidalgo M: Pancreatic cancer. N Engl J Med 362: 1605-1617, 2010.

4. Winter JM, Brennan MF, Tang LH, D'Angelica MI, Dematteo RP, Fong Y, Klimstra DS, Jarnagin WR and Allen PJ: Survival after resection of pancreatic adenocarcinoma: Results from a single institution over three decades. Ann Surg Oncol 19: 169-175, 2012.
5. Cho K, Ishiwata T, Uchida E, Nakazawa N, Korc M, Naito Z and Tajiri T: Enhanced expression of keratinocyte growth factor and its receptor correlates with venous invasion in pancreatic cancer. Am J Pathol 170: 1964-1974, 2007.

6. Cleary SP, Gryfe R, Guindi M, Greig P, Smith L, Mackenzie R, Strasberg S, Hanna S, Taylor B, Langer B and Gallinger S: Prognostic factors in resected pancreatic adenocarcinoma: Analysis of actual 5-year survivors. J Am Coll Surg 198: 722-731, 2004.

7. Galasso M, Sandhu SK and Volinia S: MicroRNA expression signatures in solid malignancies. Cancer J 18: 238-243, 2012.

8. Zhu W, Wang Y, Zhang D, Yu X and Leng X: MiR-7-5p functions as a tumor suppressor by targeting SOX18 in pancreatic ductal adenocarcinoma. Biochem Biophys Res Commun 497: 963-970, 2018.

9. Xiong J, Wang D, Wei A, Ke N, Wang Y, Tang J, He S, Hu W and Liu X: MicroRNA-410-3p attenuates gemcitabine resistance in pancreatic ductal adenocarcinoma by inhibiting HMGB1-mediated autophagy. Oncotarget 8: 107500-107512, 2017.

10. Wu Z, Zhou L, Ding G and Cao L: Overexpressions of miR-212 are associated with poor prognosis of patients with pancreatic ductal adenocarcinoma. Cancer Biomark 18: 35-39, 2017.

11. Fan Y, Shi C, Li T and Kuang T: microRNA-454 shows anti-angiogenic and anti-metastatic activity in pancreatic ductal adenocarcinoma by targeting LRP6. Am J Cancer Res 7: 139-147, 2017.

12. Chang JC and Kundranda M: Novel diagnostic and predictive biomarkers in pancreatic adenocarcinoma. Int J Mol Sci 18: E667, 2017.

13. Diab M, Muqbil I, Mohammad RM, Azmi AS and Philip PA: The role of microRNAs in the diagnosis and treatment of pancreatic adenocarcinoma. J Clin Med 5: E59, 2016.

14. Song B, Zhang C, Li G, Jin G and Liu C: MiR-940 inhibited pancreatic ductal adenocarcinoma growth by targeting MyD88. Cell Physiol Biochem 35: 1167-1177, 2015.

15. Keklikoglou I, Hosaka K, Bender C, Bott A, Koerner C, Mitra D, Will R, Woerner A, Muenstermann E, Wilhelm H, et al: MicroRNA-206 functions as a pleiotropic modulator of cell proliferation, invasion and lymphangiogenesis in pancreatic adenocarcinoma by targeting ANXA2 and KRAS genes. Oncogene 34: 4867-4878, 2015.

16. Zhang R, Leng H, Huang J, Du Y, Wang Y, Zang W, Chen X and Zhao G: miR-337 regulates the proliferation and invasion in pancreatic ductal adenocarcinoma by targeting HOXB7. Diagn Pathol 9: 171, 2014.

17. Chen C, Xue S, Zhang J, Chen W, Gong D, Zheng J, Ma J, Xue W, Chen Y, Zhai W and Zheng J: DNA-methylation-mediated repression of miR-766-3p promotes cell proliferation via targeting SF2 expression in renal cell carcinoma. Int J Cancer 141: 1867-1878, 2017.

18. Li YC, Li CF, Chen LB, Li DD, Yang L, Jin JP and Zhang B: MicroRNA-766 targeting regulation of SOX6 expression promoted cell proliferation of human colorectal cancer. Onco Targets Ther 8: 2981-2988, 2015.

19. Li X, Shi Y, Yin Z, Xue X and Zhou B: An eight-miRNA signature as a potential biomarker for predicting survival in lung adenocarcinoma. J Transl Med 12: 159, 2014.

20. Livak KJ and Schmittgen TD: Analysis of relative gene expression data using real-time quantitative PCR and the 2(-Delta Delta C(T)) method. Methods 25: 402-408, 2001.

21. Park JY, Helm J, Coppola D, Kim D, Malafa M and Kim SJ: MicroRNAs in pancreatic ductal adenocarcinoma. World J Gastroenterol 17: 817-827, 2011.

22. Mardin WA and Mees ST: MicroRNAs: Novel diagnostic and therapeutic tools for pancreatic ductal adenocarcinoma? Ann Surg Oncol 16: 3183-3189, 2009.

23. Frampton AE, Krell J, Jacob J, Stebbing J, Jiao LR and Castellano L: microRNAs as markers of survival and chemoresistance in pancreatic ductal adenocarcinoma. Expert Rev Anticancer Ther 11: 1837-1842, 2011.

24. Oh K and Lee DS: In vivo validation of metastasis-regulating microRNA-766 in human triple-negative breast cancer cells. Lab Anim Res 33: 256-263, 2017.

25. Afgar A, Fard-Esfahani P, Mehrtash A, Azadmanesh K, Khodarahmi F, Ghadir M and Teimoori-Toolabi L: MiR-339 and especially miR-766 reactivate the expression of tumor suppressor genes in colorectal cancer cell lines through DNA methyltransferase 3B gene inhibition. Cancer Biol Ther 17: 1126-1138, 2016.

26. Dittmer J: The role of the transcription factor Ets1 in carcinoma. Semin Cancer Biol 35: 20-38, 2015. 
27. Ito T, Nakayama T, Ito M, Naito S, Kanematsu T and Sekine I Expression of the ets-1 proto-oncogene in human pancreatic carcinoma. Mod Pathol 11: 209-215, 1998.

28. Lefter LP, Dima S, Sunamura M, Furukawa T, Sato Y, Abe M, Chivu M, Popescu I and Horii A: Transcriptional silencing of ETS-1 efficiently suppresses angiogenesis of pancreatic cancer. Cancer Gene Ther 16: 137-148, 2009.

29. Khanna A, Mahalingam K, Chakrabarti D and Periyasamy G: Ets-1 expression and gemcitabine chemoresistance in pancreatic cancer cells. Cell Mol Biol Lett 16: 101-113, 2011.

30. Li C, Wang Z, Chen Y, Zhou M, Zhang H, Chen R, Shi F, Wang $C$ and Rui Z: Transcriptional silencing of ETS-1 abrogates epithelial-mesenchymal transition resulting in reduced motility of pancreatic cancer cells. Oncol Rep 33: 559-565, 2015.
31. Li Z, Lin P, Gao C, Peng C, Liu S, Gao H, Wang B, Wang J, Niu J and Niu W: Integrin $\beta 6$ acts as an unfavorable prognostic indicator and promotes cellular malignant behaviors via ERK-ETS1 pathway in pancreatic ductal adenocarcinoma (PDAC). Tumour Biol 37: 5117-5131, 2016.

32. Christopher AF, Kaur RP, Kaur G, Kaur A, Gupta V and Bansal P: MicroRNA therapeutics: Discovering novel targets and developing specific therapy. Perspect Clin Res 7: 68-74, 2016. 